\title{
Anthropometric evaluation of children aged between 3 and 9 years from the Canary
} Islands

\author{
Avaliação antropométrica de crianças entre os 3 e os 9 anos de idade das ilhas Canárias
}

\author{
Cíntia Ferreira-Pêgo ${ }^{1}$, Elizabeth Galván², Silvia Morales², Joaquim Fábregas², Yazmina Martín², Nelson \\ Tavares $^{1}$ \\ ${ }^{1}$ CBIOS Lusófona's Research Center for Biosciences and Health Technologies, Av. Campo Grande 376, 1749-024 Lisbon, \\ Portugal \\ ${ }^{2}$ ICSE - Centro Integrado de Educación Superior, Calle Franchy Roca 28, 35007, Las Palmas de Gran Canaria, Spain \\ Email: nelson.tavares@ulusofona.pt
}

\begin{abstract}
Obesity was declared as a global epidemic, as it affected more and more countries worldwide. Recent evidence has shown that the rates of overweight/ obesity have reached wide-ranging proportions in children under the age of 5 years, making it one of the most serious global public health challenges of the 21 st century.

A cross-sectional analysis to assess the prevalence of normal weight, overweight, and obesity in pre-school and school children from Canary Island was designed. The final sample size analyzed included 1007 participants aged between 3 and 9 years old.

Girls were significantly more overweight and obese than boys at age 4, and more overweight, but not obese, at age 9. The odds ratio of presenting overweight or obesity were significantly higher for girls and later in childhood (in total population and both genders separately) in the fully-adjusted model. Further studies are needed to understand the behaviors leading to this condition, mainly in girls.

These results emphasize the importance of nutritional education programs, even in young children, to improve the eating choices and consequently reducing childhood obesity.
\end{abstract}

Keywords: obesity, overweight, children, Canary Island, Spain

\begin{abstract}
Resumo
A obesidade foi declarada epidemia global, já que começou a afetar cada vez mais países ao redor do mundo. As recentes evidências demostraram que as taxas de excesso de peso/obesidade atingiram elevadas proporções em crianças menores de 5 anos, tornando-o um dos mais sérios desafios de saúde pública global do século XXI.

O presente trabalho consiste numa análise transversal com o principal objetivo de avaliar a prevalência de peso normal, excesso de peso e obesidade em crianças em idade pré-escolar e escolar das Ilhas Canárias. $\mathrm{O}$ tamanho final da amostra analisada incluiu 1007 participantes com idades entre 3 e 9 anos.

As raparigas apresentaram significativamente maior excesso de peso e obesidade do que os rapazes aos 4 anos de idade, e excesso de peso, mas não obesidade, aos 9 anos. A odds ratio de excesso de peso e obesidade foi significativamente maior para as raparigas e crianças mais velhas (na população total e ambos sexos separadamente).

Estes resultados enfatizam a importância da existência de programas de educação nutricional, mesmo em crianças jovens, de forma a melhorar as escolhas alimentares e, consequentemente, reduzir a taxa de obesidade infantil.
\end{abstract}

Palavras-chave: obesidade, excesso de peso, crianças, Ilhas Canárias, Espanha 


\section{Introduction}

In 2000, the World Health Organization (WHO) warned in its annual report that obesity incidence could become an emerging public health problem (1), and in 2003 declared it as a global epidemic as it affected more and more countries worldwide (2). Rates of overweight / obesity have reached wide-ranging proportions in children under the age of 5 years (3), which became a serious global public health challenge of the $21^{\text {st }}$ century based on recent evidence. In line with this assumption, the WHO reported that more than 38 million children of this age group were classified as overweight or obese in 2017 (4). Additionally, the latest WHO report (from 2018) states that in 2016 over 340 million children aged 5-19 years had excess of weight (5). Particularly in Spain, childhood overweight and obesity is worrying considering the latest published data indicated it hadone of the highest prevalence in Europe (6). About $39 \%$ of Spanish children and adolescents, aged between 8 and 17, are overweight or obese (7) and $16.5 \%$ present abdominal obesity (8). This means that practically one in two children presents a higher weight than that recommended by WHO.

Specifically in the Canary Islands, $32.8 \%$ of individuals aged between 2 and 24 years old presented overweight, and $18 \%$ of them presented as being obese (9), placing it as the Spanish autonomous community with one of the highest rate of childhood obesity (10).

Childhood obesity is of concern not only due to the increased prevalence in worldwide populations butalso due to its multiple health consequences,including increased risk factors for cardiovascular and metabolic diseases development (11), with an impact in obesity on adulthood (12), with psychosocial consequences during childhood $(12,13)$ ultimately affecting global physical health (13). For all these reasons, the main objective of the present study was to assess the prevalence of normal weight, overweight, and obesity of a sample of children aged between 3 and 9 years old from the Canary Islands.

\section{Introdução}

Em 2000, a Organização Mundial da Saúde (OMS) alertou no seu relatório anual que a incidência de obesidade poderia vir a tornar-se um problema emergente de saúde pública (1) e, em 2003, declarou-a como uma epidemia global, já que começou a afetar cada vez mais países mundiais (2). As recentes evidências demostraram que as taxas de excesso de peso/obesidade atingiram grandes proporções em crianças menores de 5 anos (3), tornando-o um dos mais sérios desafios de saúde pública global do século XXI. De acordo com essa premissa, a OMS relatou que mais de 38 milhões de crianças dessa faixa etária foram classificadas como sofrendo de excesso de peso ou obesidade em 2017 (4). É importante ressalvar também que o último relatório da OMS de 2018 mostra que, em 2016, mais de 340 milhões de crianças de entre 5 a 19 anos de idade apresentavam excesso de peso (5).

Particularmente em Espanha, o sobrepeso e a obesidade infantil são especialmente preocupantes tendo em consideração os últimos dados publicados, sendo dos níveis mais elevados da Europa (6). Cerca de 39\% das crianças e adolescentes espanhóis, com idades entre os 8 e 17 anos, apresentam excesso de peso ou obesidade (7) e $16,5 \%$ apresentam obesidade abdominal (8). Isso significa que praticamente uma em cada duas crianças apresenta peso superior ao recomendado pela OMS. Especificamente nas Ilhas Canárias, 32,8\% dos indivíduos com idade entre os 2 e 24 anos apresentam excesso de peso, sendo $18 \%$ de obesos (9), colocando-a como a comunidade autónoma com um dos maiores índices de obesidade infantil de todo o território espanhol (10). A obesidade infantil é extremamente preocupante não só apenas devido ao aumento da prevalência da doença em vários países, mas também devido às múltiplas consequências para a saúde a curto, médio e longo prazo, como o aumento dos fatores de risco para o desenvolvimento de doenças cardiovasculares e metabólicas (11), aumentando da possibilidade de apresentar obesidade na idade adulta (12), levando à presença de consequências psicossociais na infância $(12,13)$, afetando finalmente a saúde física no geral (13). Por todas essas razões, o principal objetivo do presente estudo foi avaliar a prevalência de peso normal, excesso de peso e obesidade de uma amostra de crianças de 3 a 9 anos de idade das ilhas Canárias. 


\section{Design and study population}

The present study is a cross-sectional analysis designed to assess the prevalence of normal weight, overweight, and obesity in pre-school and school children from Canary Island.

The participants aged between 3 and 9 years and were recruited from private schools from Gran Canaria island. The children's inclusion criteria involved written parental consent (following the Declaration of Helsinki), not being sick and being present on the scheduled visit day. The evaluations took place during the 2018/2019 school year. The effective sample size of the present work was 1007 participants.

\section{Anthropometric parameters assessment}

Trained personnel measured weight using an electronic scale, recording the value to the nearest decigram $(0.1$ $\mathrm{kg}$ ), and height using a calibrated stadiometer. Stature was assessed with the children standing upright, with their backs to the stadiometer and with their feet and knees together, their head positioned so that the gaze remained horizontal, according to the Frankfurt plane (16). The value was recorded to the nearest millimeter $(0.1 \mathrm{~cm})$. All the participants wore light clothing and no shoes during the measurements. BMI was afterward calculated from the measurements obtained in each child using the normalized formula [Weight (in $\mathrm{kg}$ )/ Height $^{2}$ (in m)] (14). Normal weight, overweight and obesity were classified using the international age and gender cut-off points according to those proposed by the International Obesity Task Force (IOTF) (15).

\section{Statistical analysis}

Data are presented either as means and standard deviation (SD) for continuous variables or numbers and percentages for dichotomous variables. We compared the distribution of the selected characteristics between groups using Pearson $\chi^{2}$ tests for categorical variables or Student's t-tests or analysis of variance (ANOVA), as appropriate, for continuous variables. Logistical regression models were fitted to assess the associations between presenting BMI $\geq 25 \mathrm{~kg} / \mathrm{m}^{2}$ (dependent variable) and gender (two categories), age (years) or age categories (seven categories) as exposure. The models were adjusted for gender, age, and school origin, except when any of these variables were the independent variable. All statistical tests were two-tailed and the significance level was set at $\mathrm{p}<0.05$. All analyses were

\section{Material e Métodos}

\section{Desenho e estudo da população}

O presente estudo é uma análise transversal com o objetivo de avaliar a prevalência de peso normal, excesso de peso e obesidade em crianças de idade pré-escolar e escolar das Ilhas Canárias.

Os participantes com idades entre os 3,00 e 9,99 anos foram recrutados em escolas de ensino privado da ilha de Gran Canaria. Nos critérios de inclusão das crianças constavam o consentimento por escrito dos pais (de acordo com a Declaração de Helsínquia), não apresentar doença aparente e estar presente no dia agendado para a visita. As avaliações ocorreram durante o ano letivo de 2018/2019. O tamanho efetivo da amostra do presente trabalho foi de 1007 participantes.

\section{Avaliação dos dados antropométricos}

O peso foi medido por profissionais treinados utilizando uma balança eletrónica, registrando o valor no decigrama mais próximo $(0,1 \mathrm{~kg})$ e a altura usando um estadiómetro calibrado. A estatura foi avaliada com as crianças em pé, de costas para o estadiómetro e com os pés e joelhos juntos, a cabeça posicionada para que o olhar permanecesse horizontal, de acordo com o plano de Frankfurt (16). O valor foi registado no milímetro mais próximo $(0,1 \mathrm{~cm})$. Todos os participantes usaram roupas leves e não usaram sapatos durante todas as medições. O Índice de Massa Corporal (IMC) foi posteriormente calculado a partir das medidas obtidas em cada criança, utilizando a fórmula normalizada [Peso (em kg)/Altura ${ }^{2}$ (em m)] (14). Peso normal, excesso de peso e obesidade foram classificados usando os pontos de corte internacionais de idade e sexo, de acordo com os propostos pelo International Obesity Task Force (IOTF) (15).

\section{Análise estatística}

Os dados são apresentados como médias e desvio padrão (DP) para variáveis contínuas ou números (n) e percentagens (\%) para variáveis dicotómicas. Comparámos a distribuição das características selecionadas entre os grupos usando testes de Pearson $\chi 2$ para variáveis categóricas ou testes $t$ de Student ou análise de variância (ANOVA), conforme apropriado, para variáveis contínuas. Os modelos de regressão logística foram ajustados para avaliar as associações entre apresentar excesso de peso e obesidade (variável dependente) e sexo (duas categorias), idade (anos) ou categorias de idade (sete categorias) como exposição. Os modelos foram ajustados para sexo, idade e proveniência escolar, exceto quando qualquer dessas variáveis fosse 
performed using the SPSS software version 22.0 (SPSS Inc, Chicago, IL).

\section{Results}

A total of 1007 children from the Canary Islands (522 boys and 485 girls) with a mean age of 6.65 (SD:1.94) years took part in the present analysis. The general characteristics of the studied population according to gender are summarized in Table 1. Statistical differences were found between both genders regarding height and weight, however, this significant variance disappeared when BMI was calculated. a variável independente. Todos os testes estatísticos foram bicaudais e o nível de significância foi estabelecido em $\mathrm{P}$ $<0,05$. Todas as análises foram realizadas utilizando o software SPSS versão 22.0 (SPSS Inc, Chicago, IL).

\section{Resultados}

Um total de 1007 crianças das Ilhas Canárias (522 rapazes e 485 raparigas), com idade média de 6,65 anos (DP: 1,94), participaram nesta análise. As características gerais da população estudada de acordo com o sexo estão resumidas na Tabela 1. Foram encontradas diferenças estatísticas entre os sexos em relação à estatura e peso, no entanto, essa variação significativa desapareceu quando o cálculo do IMC foi levado a cabo.

Table 1/ Tabela 1 - General characteristics of the study population categorized by gender./ Tabela 1. Características gerais da população estudada categorizada por sexo.

\begin{tabular}{|c|c|c|c|c|}
\hline & $\begin{array}{l}\text { All population/ } \\
\text { População total } \\
\quad(\mathrm{n}=1007)\end{array}$ & $\begin{array}{c}\text { Boys/ } \\
\text { Rapazes } \\
(n=522)\end{array}$ & $\begin{array}{c}\text { Girls/ } \\
\text { Raparigas } \\
(\mathrm{n}=485)\end{array}$ & $\begin{array}{c}\mathrm{p}- \\
\text { value }^{\mathrm{a}}\end{array}$ \\
\hline Age/ Idade, years/ anos & $6.65(1.94)$ & $6.73(1.98)$ & $6.57(1.91)$ & 0.185 \\
\hline Height/ Altura, m & $1.22(0.14)$ & $1.23(0.14)$ & $1.21(0.13)$ & 0.005 \\
\hline Weight/ Peso, kg & $26.06(8.67)$ & $26.66(9.15)$ & $25.39(8.09)$ & 0.020 \\
\hline $\mathrm{BMI} / \mathrm{IMC}, \mathrm{kg} / \mathrm{m}^{2}$ & $17.11(2.96)$ & $17.16(3.08)$ & $17.05(2.82)$ & 0.574 \\
\hline \multicolumn{5}{|c|}{$\begin{array}{l}\text { Age categories/ Categorias de idade, } \\
\%(n)\end{array}$} \\
\hline 3 years/ anos & $10.96(111)$ & $11.24(59)$ & $10.66(52)$ & \\
\hline 4 years/ anos & $12.54(127)$ & $11.62(61)$ & $13.52(66)$ & \\
\hline 5 years/ anos & $11.65(118)$ & $11.82(62)$ & $11.48(56)$ & \\
\hline 6 years/ anos & $15.99(162)$ & $14.86(78)$ & $17.21(84)$ & 0.618 \\
\hline 7 years/ anos & $16.58(168)$ & $15.81(83)$ & $17.42(85)$ & \\
\hline 8 years/ anos & $17.87(181)$ & $18.28(96)$ & $17.42(85)$ & \\
\hline 9 years/ anos & $13.82(140)$ & $15.80(83)$ & $11.68(57)$ & \\
\hline
\end{tabular}

Data expressed as means (SD) or percentages (n). Abbreviations: BMI, body mass index. a P values for comparisons between groups were tested by Student's t-test or Pearson $\chi 2$ as appropriate./ Dados expressos em média (DP) ou percentagens (n). Abreviaturas: IMC, Indice de Massa Corporal. a Valores P para comparações entre grupos foram testados mediante teste $t$ de Student ou Pearson $\chi 2$ como apropriado. 
Table 2/ Tabela 2 - Anthropometric measurements according to gender and age categories./ Dados antropométricos de acordo com o sexo e categorias de idade.

\begin{tabular}{|c|c|c|c|c|}
\hline & $\begin{array}{l}\text { All population/ } \\
\text { População total }\end{array}$ & Boys/ Rapazes & Girls/ Raparigas & $\mathrm{p}$-value \\
\hline 3 years/ anos & $(n=111)$ & $(n=59)$ & $(n=52)$ & \\
\hline Height/ Altura & $1.01(0.06)$ & $1.01(0.05)$ & $1.01(0.08)$ & 0.935 \\
\hline Weight/ Peso & $16.57(2.44)$ & $16.75(2.14)$ & $16.37(2.75)$ & 0.410 \\
\hline BMI/ IMC & $16.20(1.57)$ & $16.39(1.44)$ & 15.97 (1.69) & 0.155 \\
\hline 4 years/ anos & $(n=127)$ & $(n=61)$ & $(n=66)$ & \\
\hline Height/ Altura & $1.08(0.08)$ & $1.09(0.05)$ & $1.08(0.09)$ & 0.720 \\
\hline Weight/ Peso & $18.70(2.96)$ & $18.71(2.56)$ & $18.68(3.30)$ & 0.953 \\
\hline BMI/ IMC & $16.01(2.35)$ & $15.88(1.76)$ & $16.14(2.80)$ & 0.535 \\
\hline 5 years/ anos & $(n=118)$ & $(n=62)$ & $(n=56)$ & \\
\hline Height/ Altura & $1.14(0.07)$ & $1.16(0.07)$ & $1.13(0.06)$ & 0.014 \\
\hline Weight/ Peso & $21.94(4.67)$ & $22.43(4.86)$ & $21.40(4.42)$ & 0.233 \\
\hline $\mathrm{BMI} / \mathrm{IMC}$ & $16.59(2.50)$ & $16.53(2.60)$ & $16.65(2.41)$ & 0.785 \\
\hline 6 years/ anos & $(n=162)$ & $(n=78)$ & $(n=84)$ & \\
\hline Height/ Altura & $1.21(0.06)$ & $1.22(0.06)$ & $1.20(0.06)$ & 0.028 \\
\hline Weight/ Peso & $24.23(5.12)$ & $24.53(5.61)$ & $23.95(4.63)$ & 0.476 \\
\hline BMI/ IMC & $16.48(2.51)$ & $16.40(2.53)$ & $16.55(2.51)$ & 0.699 \\
\hline 7 years/ anos & $(n=168)$ & $(n=83)$ & $(n=85)$ & \\
\hline Height/ Altura & $1.27(0.06)$ & $1.27(0.05)$ & $1.26(0.06)$ & 0.172 \\
\hline Weight/ Peso & $28.77(6.11)$ & $29.41(6.30)$ & $28.14(5.88)$ & 0.179 \\
\hline BMI/ IMC & $17.80(3.01)$ & $18.02(3.22)$ & $17.57(2.79)$ & 0.337 \\
\hline 8 years/ anos & $(\mathrm{n}=181)$ & $(n=96)$ & $(n=85)$ & \\
\hline Height/ Altura & $1.32(0.07)$ & $1.33(0.07)$ & $1.30(0.07)$ & 0.013 \\
\hline Weight/ Peso & $30.72(7.39)$ & $31.51(8.17)$ & $29.81(6.33)$ & 0.123 \\
\hline $\mathrm{BMI} / \mathrm{IMC}$ & $17.61(3.34)$ & $17.70(3.72)$ & $17.50(2.87)$ & 0.682 \\
\hline 9 years/ anos & $(n=140)$ & $(n=83)$ & $(n=57)$ & \\
\hline Height/ Altura & $1.39(0.07)$ & $1.39(0.07)$ & $1.38(0.07)$ & 0.469 \\
\hline Weight/ Peso & $35.92(8.98)$ & $35.74(9.41)$ & 36.18 (8.39) & 0.778 \\
\hline BMI/ IMC & $18.47(3.56)$ & $18.26(3.64)$ & $18.79(3.45)$ & 0.389 \\
\hline
\end{tabular}

Data expressed as means (SD). Height expressed in meters (m), weight in kilograms $(\mathrm{kg})$ and BMI in $\mathrm{kg} / \mathrm{m} 2$. Abbreviations: BMI, body mass index. a $\mathrm{P}$ values for comparisons between groups were tested by Student's t-test./ Dados expresso como media (DP). Altura expressa em metros (m), peso em quilogramas $(\mathrm{kg})$ e IMC em $\mathrm{kg} / \mathrm{m} 2$. Abreviaturas: IMC, Indice de Massa Corporal. a Valores P para comparações entre grupos foram testados mediante teste t de Student. 
Table 2 shows the anthropometric measurements for each age categories assessed, separated by gender. Boys were statistically taller than girls at ages 5, 6, and 8 . No other differences were observed in additional parameters or age categories.

Girls were significantly more overweight and obese than boys at age $4(\mathrm{p}=0.034)$. Results, also observed in Table 3, indicate that girls at age 9 were more overweight than boys with the same age. However, boys were more obese $(15.70 \%)$ than girls $(10.50 \%)$ at the same age. Differences $(p=0.023)$ were also found in total population (not subdivided by age categories) when the analysis was performed taking into consideration overweight and obesity in the same category (BMI $\left.\geq 25 \mathrm{~kg} / \mathrm{m}^{2}\right)$, demonstrating that girls $(30.90 \%)$ were heavier than boys $(25.10 \%)$ (data not shown).
A Tabela 2 mostra as medidas antropométricas para cada faixa etária avaliada, separadas por sexo. Os rapazes demonstraram ser estatisticamente mais altos que as raparigas aos 5, 6 e 8 anos de idade. Nenhuma outra diferença foi observada nos restantes parâmetros ou categorias de idade.

As raparigas demonstraram apresentar significativamente maior excesso de peso e obesidade do que os rapazes aos 4 anos $(p=0,034)$. Outros resultados também observados na Tabela 3 indicaram que as crianças do sexo feminino com 9 anos apresentaram mais excesso de peso do que as crianças do sexo masculino com a mesma idade. No entanto, os rapazes foram mais obesos $(15,70 \%)$ do que as raparigas $(10,50 \%)$ na mesma idade. Também foram encontradas diferenças $(\mathrm{p}=0,023)$ na população total (não subdivididas por categorias de idade) quando a análise foi realizada considerando-se o excesso de peso e obesidade na mesma categoria, demonstrando que as raparigas $(30,90 \%)$ eram mais pesadas que os rapazes $(25,10 \%)$ (dados não mostrados).

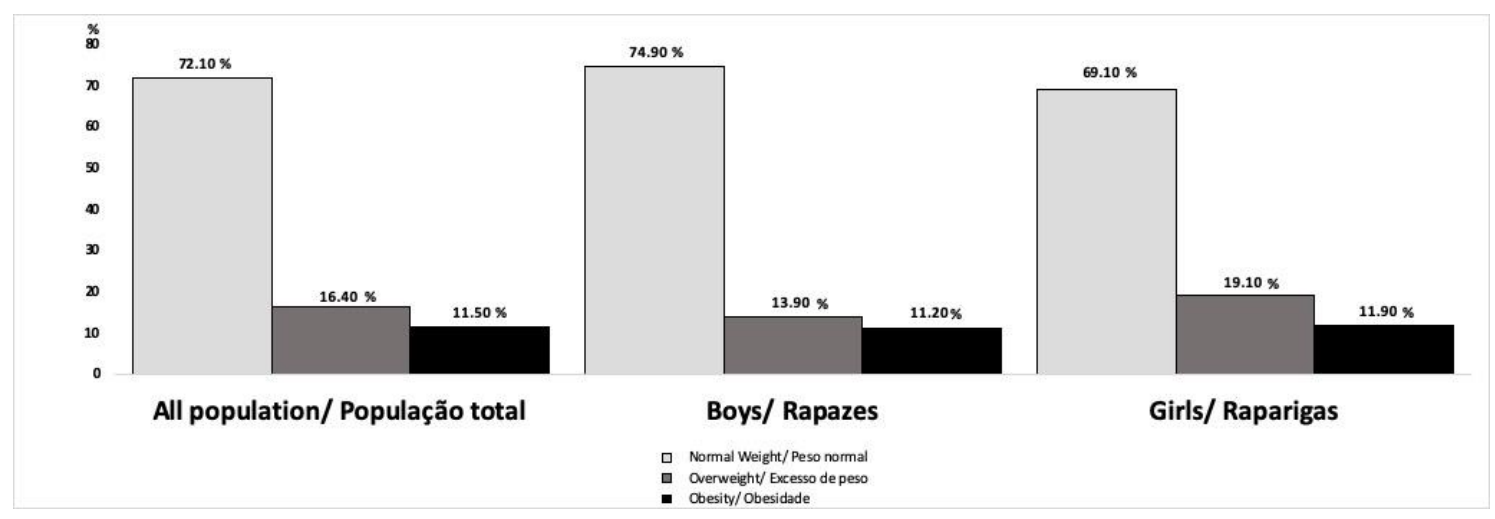

Figure 1/ Figura 1 - Percentage of individuals presenting normal weight, overweight and obesity, according to IOTF criteria categorized by gender/ Percentagem de indivíduos apresentando peso normal, excesso de peso ou obesidade de acordo comos critérios da IOTF categorizada por sexo. 
Table 3/ Tabela 3 - Prevalence of normal weight, overweight and obesity according gender and age categories./. Prevalência de peso normal, excesso de peso e obesidade de acordo com o sexo e categorias de idade.

\begin{tabular}{|c|c|c|c|c|}
\hline & $\begin{array}{c}\text { All } \\
\text { population/ } \\
\text { População } \\
\text { total }\end{array}$ & $\begin{array}{c}\text { Boys/ } \\
\text { Rapazes }\end{array}$ & $\begin{array}{c}\text { Girls/ } \\
\text { Raparigas }\end{array}$ & $\begin{array}{c}\text { p- } \\
\text { value }^{a}\end{array}$ \\
\hline \multicolumn{5}{|l|}{3 years/ anos } \\
\hline Normal Weight/ Peso normal & $86.50(96)$ & $86.40(51)$ & $86.50(45)$ & \\
\hline Overweight/ Excesso de peso & $9.90(11)$ & $10.20(6)$ & $9.60(5)$ & 0.988 \\
\hline Obesity/ Obesidade & $3.60(4)$ & $3.40(2)$ & $3.80(2)$ & \\
\hline \multicolumn{5}{|l|}{4 years/ anos } \\
\hline Normal Weight/ Peso normal & $81.10(103)$ & $88.50(54)$ & $74.20(49)$ & \\
\hline Overweight/ Excesso de peso & $11.00(14)$ & $9.80(6)$ & $12.10(8)$ & 0.034 \\
\hline Obesity/ Obesidade & $7.90(10)$ & $1.60(1)$ & $13.60(9)$ & \\
\hline \multicolumn{5}{|l|}{5 years/ anos } \\
\hline Normal Weight/ Peso normal & $69.50(82)$ & $75.80(47)$ & $62.50(35)$ & \\
\hline Overweight/ Excesso de peso & $16.90(20)$ & $12.90(8)$ & $21.40(12)$ & 0.285 \\
\hline Obesity/ Obesidade & $13.60(16)$ & $11.30(7)$ & $16.10(9)$ & \\
\hline \multicolumn{5}{|l|}{6 years/ anos } \\
\hline Normal Weight/ Peso normal & $74.70(121)$ & $79.50(62)$ & $70.20(59)$ & \\
\hline Overweight/ Excesso de peso & $14.20(23)$ & $12.80(10)$ & $15.50(13)$ & 0.325 \\
\hline Obesity/ Obesidade & $11.10(18)$ & $7.70(6)$ & $14.30(12)$ & \\
\hline \multicolumn{5}{|l|}{7 years/ anos } \\
\hline Normal Weight/ Peso normal & $62.50(105)$ & $61.40(51)$ & $63.50(54)$ & \\
\hline Overweight/ Excesso de peso & $19.00(32)$ & $18.10(15)$ & $20.00(17)$ & 0.788 \\
\hline Obesity/ Obesidade & $18.50(31)$ & $20.50(17)$ & $16.50(14)$ & \\
\hline \multicolumn{5}{|l|}{8 years/ anos } \\
\hline Normal Weight/ Peso normal & $70.70(128)$ & $69.80(67)$ & $71.80(61)$ & \\
\hline Overweight/ Excesso de peso & $19.30(35)$ & $17.70(17)$ & $21.20(18)$ & 0.439 \\
\hline Obesity/ Obesidade & $9.90(18)$ & $12.50(12)$ & $7.10(6)$ & \\
\hline \multicolumn{5}{|l|}{9 years/ anos } \\
\hline Normal Weight/ Peso normal & $65.00(91)$ & $71.10(59)$ & $56.10(32)$ & \\
\hline Overweight/ Excesso de peso & $21.40(30)$ & $13.30(11)$ & $33.30(19)$ & 0.017 \\
\hline Obesity/ Obesidade & $13.60(19)$ & $15.70(13)$ & $10.50(6)$ & \\
\hline \multicolumn{5}{|l|}{ TOTAL } \\
\hline Normal Weight/ Peso normal & $72.10(726)$ & $74.90(391)$ & $69.10(335)$ & \\
\hline Overweight/ Excesso de peso & $16.40(165)$ & $14.00(73)$ & $19.00(92)$ & 0.076 \\
\hline Obesity/ Obesidade & $11.50(116)$ & $11.10(58)$ & $12.00(58)$ & \\
\hline
\end{tabular}

Data expressed as percentages (n). a $\mathrm{P}$ values for comparisons between groups were tested by Pearson $\chi 2$. b Normal weight, overweight or obesity were categorized according to IOTF criteria (15)./ Dados expressos como percentagem (n). a Valores P para comparações entre grupos foram testados mediante Pearson $\chi 2$. b Peso normal, excesso de peso e obesidade foram categorizados de acordo com os critérios da IOTF (15). 
As shown in Figure 1, within the entire studied population the prevalence of overweight was $16.40 \%$ and obesity of $11.50 \%$. Girls were not statistically more overweight $(19.00 \%)$ and obese $(12.00 \%)$ than boys ( $14.00 \%$ and $11.10 \%$, respectively).

The odds ratio of presenting BMI higher than $25 \mathrm{~kg} / \mathrm{m}^{2}$ (Table 4) were significantly higher for girls and later in childhood (in the total studied population and both genders separately) in the fully-adjusted model. Participants aged 5, 6, 7, 8 and 9 years presented a significantly higher risk of being overweight or obese when 3 year old children were considered as reference. Similar results were observed when only girls were analyzed, except for 8 years category. In the same line, boys analyzed individually at 7,8 and 9 years of age showed a statistically higher risk of presenting BMI over $25 \mathrm{~kg} /$ $\mathrm{m}^{2}$.
Como mostra a Figura 1, a prevalência de excesso de peso na população total foi de $16,40 \%$ e obesidade de $11,50 \%$. As raparigas apresentaram (não estatisticamente) maior excesso de peso $(19,00 \%)$ e obesidade $(12,00 \%)$ do que os rapazes $(14,00 \%$ e $11,10 \%$, respetivamente).

O odds ratio de apresentar IMC excesso de peso e obesidade (Tabela 4) foi significativamente maior para crianças do sexo feminino e crianças mais velhas (na população total e em ambos os sexos separadamente) no modelo mais ajustado. Participantes de 5, 6, 7, 8 e 9 anos apresentaram um risco significativamente maior de estar acima do peso recomendado, em comparação com as crianças de 3 anos. Resultados semelhantes foram observados quando apenas as raparigas foram analisadas, com exceção da categoria de 8 anos. Na mesma linha, quando os rapazes foram analisados individualmente, as categorias de 7, 8 e 9 anos apresentaram um risco estatisticamente maior de apresentar excesso de peso e obesidade, tendo em consideração os rapazes de 3 anos como referência.

Table 4/ Tabela 4 - Association (odds ratio) between presenting BMI $>25 \mathrm{~kg} /$ $\mathrm{m} 2$ and gender or age categories./ Associação (odds ratio) entre apresentar IMC $>25 \mathrm{~kg} / \mathrm{m} 2$ e sexo ou categorias de idade.

\begin{tabular}{|c|c|c|c|}
\hline Variables/Variáveis & $\begin{array}{l}\text { All population/ } \\
\text { População total } \\
\quad(n=1007)\end{array}$ & $\begin{array}{l}\text { Boys/ Rapazes } \\
\quad(\mathrm{n}=522)\end{array}$ & $\begin{array}{l}\text { Girls/ Raparigas } \\
\qquad(\mathrm{n}=485)\end{array}$ \\
\hline Gender/Sexo ${ }^{a}$ & $1.38(1.04-1.83)$ & - & - \\
\hline Age/ Idade & $1.20(1.11-1.29)$ & $1.23(1.10-1.37)$ & $1.16(1.05-1.29)$ \\
\hline \multicolumn{4}{|c|}{$\begin{array}{l}\text { Age categories/ Categorias de } \\
\text { idade }\end{array}$} \\
\hline 3 years/ anos & 1.00 (ref.) & 1.00 (ref.) & 1.00 (ref.) \\
\hline 4 years/ anos & $1.50(0.74-3.03)$ & $0.84(0.28-2.48)$ & $2.21(0.83-5.87)$ \\
\hline 5 years/ anos & $2.75(1.40-5.39)$ & $2.02(0.78-5.19)$ & $3.73(1.41-9.83)$ \\
\hline 6 years/ anos & $2.13(1.11-4.08)$ & $1.62(0.64-4.10)$ & $2.70(1.06-6.86)$ \\
\hline 7 years/ anos & $3.74(1.99-7.03)$ & $3.97(1.66-9.46)$ & $3.48(1.39-8.70)$ \\
\hline 8 years/ anos & $2.69(1.43-5.08)$ & $2.76(1.16-6.57)$ & $2.50(0.99-6.36)$ \\
\hline 9 years/ anos & $3.64(1.90-6.99)$ & $2.65(1.09-6.46)$ & $4.98(1.90-13.10)$ \\
\hline
\end{tabular}

Data expressed as ORs (95\% Confidence Interval). a Gender was coded as 0 for boys and 1 for girls. Logistic regression model adjusted for gender, age and school origin (except when gender or age were the independent variable)./ Dados expressos em OR (95\% intervalo de confiança). a Sexo foi codificado como 0 para rapazes e 1 para raparigas. Modelo de regressão logística ajustada por sexo, idade e colégio (exceto quando sexo ou idade eram as variáveis independentes). 


\section{Discussion}

The results of the present study indicate that girls and older childhood were more likely to be overweight or obese. In girls, each age category after 5 years is associated with presenting BMI $\geq 25 \mathrm{~kg} / \mathrm{m}^{2}$. Boys older than 7 years presented significantly more risk of higher weight than recommended.

The total prevalence of obesity and overweight observed in the present analysis was similar to those published in 2012 (6). However, more recently published results (2019) showed obesity prevalence values very low compared to ours (3). Still, the overweight prevalence was very similar to those published in the present document. These differences can be explained by the fact that Cadenas-Sanchez and coworkers assessed all the Spanish territory meanwhile we only evaluated Canary Island, which, as described previously, is the Spanish autonomous community with a higher prevalence of childhood obesity.

Taking into consideration age comparison, to the best of our knowledge, the present work is the first one to describe the relationship between age categories and the risk of overweight and obesity in Canary Island preschool and school children.

Regarding gender evaluation, the results published until now are not so clear, since it seems to depend on the reference cut point used and also the type of population assessed. A recent article, confirming our results, found that girls are more overweight than boys in school-age (3). However, other older publications found that the overall prevalence of overweight, and obesity as well, was higher in boys than in girls $(6,9,10)$. This seems to indicate a change in gender tendencies, and that while in the past the boys were heavier, now we need to pay more attention to girls. It was not the objective of the present work to evaluate these variables, thus further studies are needed to understand the behaviors leading to this condition. To the best of our knowledge, the present study is the first one to assess exclusively anthropometric data in children aged between 3 and 9 years old. These results emphasize the importance of nutritional education programs, even in young age, to improve the eating choices and consequently reducing childhood obesity. In this stage of life overweight is not associated with higher mortality rates in the short term, however, the risk increases in adult life. Follow-up studies in children and adolescents show that the likelihood of being obese in adulthood is twofold in obese children compared to the non-obese (10).

\section{Discussão}

Os resultados do presente estudo indicam que ser rapariga e ser mais velho apresentaram maior probabilidade de estar acima do peso recomendado. Após os 5 anos de idade, cada ano cumprido nas raparigas está associado a apresentar excesso de peso. Rapazes com mais de 7 anos apresentaram um risco significativamente maior de ter peso superior ao recomendado.

A prevalência total de obesidade e excesso de peso observada na presente análise foi semelhante à publicada em 2012 (6). No entanto, outros resultados publicados no presente ano de 2019 mostraram valores de prevalência de obesidade muito mais baixos em comparação com os observados por nós (3). Ainda assim, a prevalência de excesso de peso foi muito semelhante à publicada no presente documento. Essas diferenças podem ser explicadas pelo facto de os autores do trabalho avaliarem todo o território espanhol, enquanto nós somente avaliámos as Ilhas Canárias, que tal como descrito anteriormente, é a comunidade autónoma espanhola com maior prevalência de obesidade infantil.

Tendo em consideração a comparação etária, tanto quanto sabemos, o presente trabalho é o primeiro a descrever a relação entre categorias etárias e o risco de excesso de peso e obesidade em crianças pré-escolares e escolares das Ilhas Canárias.

Em relação à avaliação por sexo, os resultados publicados até o momento não são claros, pois parecem depender do ponto de corte de referência utilizado, assim como do tipo de população avaliada. Um artigo recente, que vem confirmar os nossos resultados, constatou que as raparigas têm um maior excesso de peso que os rapazes em idade escolar (3). No entanto, outros trabalhos anteriores relataram que a prevalência geral de excesso de peso e obesidade foi maior em rapazes do que em raparigas $(6,9,10)$. Esta evidência parece indicar que a tendência sobre o sexo mudou, já que no passado os rapazes eram mais pesados, contudo, parece que agora precisamos de prestar mais atenção às raparigas. Não era o objetivo do presente trabalho avaliar estas variáveis explicativas, portanto e por esse motivo, serão necessários futuros estudos para esclarecer os comportamentos que podem estar a levar a esta condição. Pelo que sabemos, o presente estudo é o primeiro a avaliar exclusivamente dados antropométricos em crianças com idades compreendidas entre os 3 e 9 anos. Estes resultados enfatizam a importância de programas de educação nutricional, mesmo em crianças de curta idade, de forma a melhorar as suas escolhas alimentares e, consequentemente, reduzir as taxas de obesidade infantil. Estudos de acompanhamento em crianças e 


\section{Conclusion}

Girls showed a significantly higher prevalence of overweight and obesity than boys at age 4 and 9 . Individuals over 5 years old showed a higher risk of being overweight or obese at each age category. Similar results were observed for boys and girls separately. Being a girl or being in later childhood was related to presenting $\mathrm{BMI} \geq 25 \mathrm{~kg} / \mathrm{m}^{2}$.

\section{Acknowledgements}

The authors acknowledge all the collaborators and participants involved in the study.

\section{Conflict of interest}

All of the authors declare that there is no financial or personal relationship that can be understood as representing a potential conflict of interest. adolescentes mostram que a probabilidade de apresentar obesidade em adultos é dupla em crianças obesas em comparação com as não obesas (10). É importante ressalvar de que durante a infância, o excesso de peso não está associado a maiores taxas de mortalidade a curto prazo, no entanto, aumenta este risco durante a vida adulta.

\section{Conclusão}

As meninas apresentaram uma prevalência significativamente maior de sobrepeso e obesidade do que os meninos de 4 e 9 anos. Indivíduos com mais de 5 anos apresentaram maior risco de estar com sobrepeso ou obesidade, em cada categoria de idade. Resultados semelhantes foram observados para meninos e meninas separadamente. Ser criança do sexo feminino e idosos estavam relacionados a apresentar IMC $>25 \mathrm{~kg} / \mathrm{m}^{2}$.

\section{Agradecimentos}

Os autores agradecem a todos os colaboradores e participantes envolvidos no estudo.

\section{Conflito de Interesses}

Todos os autores declaram que não há relacionamento financeiro ou pessoal que possa ser entendido como representando um potencial conflito de interesses. 


\section{References/ Referências}

1. World Health Organization. Obesity: Preventing and Managing the Global Epidemic - WHO Technical Report Series. WHO Technical Report Series. 2000

2. Gurr MI. Diet, nutrition and the prevention of chronic diseases (WHO, 1990). Eur J Clin Nutr. 1991;45(12):619-61923.

3. Cadenas-Sanchez C, Intemann T, Labayen I, Artero EG, Alvarez-Bueno C, Sanchis-Moysi J, et al. Prevalence of severe/morbid obesity and other weight status and anthropometric reference standards in Spanish preschool children: The PREFIT project. Pediatr Res. 2019 Feb 18;

4. World Health Organization. WHO $\mid$ Childhood overweight and obesity. WHO. World Health Organization; 2017.

5. Mamrot P, Hanć T. The Association of the Executive Functions with Overweight and Obesity Indicators in Children and Adolescents: A Literature Review. Neurosci Biobehav Rev. 2019 Dec 1;107:59-68.

6. Valdés-Pizarro J, Royo-Bordonada M. Prevalence of childhood obesity in Spain: National Health Survey 2006-2007. Nutr Hosp. 2012;27(1):154-60.

7. Sánchez-Cruz J-J, Jiménez-Moleón JJ, Fernández-Quesada F, Sánchez MJ. Prevalence of Child and Youth Obesity in Spain in 2012. Rev Española Cardiol (English Ed. 2013 May 1;66(5):371-6.

8. Schröder H, Ribas L, Koebnick C, Funtikova A, Gomez SF, Fíto M, et al. Prevalence of abdominal obesity in Spanish children and adolescents. do we need waist circumference measurements in pediatric practice? Alemany M, editor. PLoS One. 2014 Jan 27;9(1):e87549.

9. Serra Majem L, Peña Quintana L, Ribas Barba L, Aranceta Bartrina J, Pérez Rodrigo C, Saavedra Santana P. Childhood and adolescent obesity in Spain Results of the enKid study (1998-2000). Med Clin (Barc). 2003 Jan;121(19):725-32.

10. Pérez-Rodrigo C, Bartrina JA, Majem LS, Moreno B, Rubio AD. Epidemiology of obesity in Spain. Dietary guidelines and strategies for prevention. In: International Journal for Vitamin and Nutrition Research. 2006. p. 163-71.

11. del Río NG, González-González CS, Martín-González R, Navarro-Adelantado V, Toledo-Delgado P, García-Peñalvo F. Effects of a Gamified Educational Program in the Nutrition of Children with Obesity. J Med Syst. 2019 Jul 22;43(7):198.

12. Luppino FS, De Wit LM, Bouvy PF, Stijnen T, Cuijpers P, Penninx BWJH, et al. Overweight, obesity, and depression: A systematic review and metaanalysis of longitudinal studies. Vol. 67, Archives of General Psychiatry. American Medical Association; 2010. p. 220-9.

13. Bhadoria A, Sahoo K, Sahoo B, Choudhury A, Sufi N, Kumar R. Childhood obesity: Causes and consequences. J Fam Med Prim Care. $2015 ; 4(2): 187$.

14. Wijnhoven TM, Van Raaij JM, Spinelli A, Starc G, Hassapidou M, Spiroski I, et al. WHO European Childhood Obesity Surveillance Initiative: Body mass index and level of overweight among 6-9-year-old children from school year 2007/2008 to school year 2009/2010. BMC Public Health. 2014 Dec $7 ; 14(1): 806$

15. Cole TJ, Bellizzi MC, Flegal KM, Dietz WH. Establishing a standard definition for child overweight and obesity worldwide: international survey. BMJ. 2000;320.

16. Madden A.M., Tsikoura T., Stott D.J. The estimation of body height from ulna length in healthy adults from different ethnic groups. J. Hum. Nutr. Diet. 2012: $25,121-128$. 